\title{
La reconsideración de la ira como emoción política. Sobre Anger and Forgiveness de Martha Nussbaum
}

\author{
[Reconsidering Anger as a Political Emotion: On Martha Nussbaum's Anger \\ and Forgiveness]
}

\author{
MARÍA JiMENA SÁENZ \\ Consejo Nacional de Investigaciones Científicas y Técnicas \\ Universidad Nacional de La Plata \\ mjimenasaenz@hotmail.com
}

\begin{abstract}
Resumen: Esta nota crítica analiza la perspectiva que Martha Nussbaum presenta sobre la emoción de la ira en su último libro Anger and Forgiveness. Resentment, Generosity, Justice (2016). Para ello sitúo esta obra en el contexto del proyecto filosófico de la autora y señalo algunos cambios y continuidades en su análisis de la ira; después reviso a la luz de este nuevo libro algunas de las críticas que, centradas en la reivindicación de la ira, ha recibido su propuesta de una cultura política centrada en las emociones "humanizadoras".

Palabras clave: emociones, filosofía, cultura política, empatía, compasión
\end{abstract}

\begin{abstract}
This critical note examines Martha Nussbaum's perspective on anger presented in her last book Anger and Forgiveness. Resentment, Generosity, Justice (2016). I place this book in the context of Nussbaum's philosophical project in order to trace changes and continuities in her views on anger, and then I examine some criticisms that have been held against her proposal of a political culture based on humanizing emotions based on the vindication of anger.
\end{abstract}

Key words: emotions, Philosophy, political culture, empathy, compassion

El último libro de la filósofa norteamericana Martha Nussbaum ${ }^{1}$ se ocupa de la ira, así como del perdón como su posible contraparte y del lugar que debe tener esa emoción en las interacciones humanas en las sociedades democráticas. El análisis de la ira se desarrolla en dos partes, que tienen, cada una, un peso diferente en la obra; una de ellas se dedica a señalar los problemas intrínsecos de la ira, mientras que la otra expone sus efectos negativos en la comunidad política.

${ }^{1}$ M.C. Nussbaum, Anger and Forgiveness. Resentment, Generosity, Justice, Oxford University Press, Nueva York, 2016. Hay que señalar también que recientemente Nussbaum publicó, en coautoría con S. Levmore, Aging Thoughtfully. Conversations about Retirement, Romance, Wrinkles, and Regret, Oxford University Press, Oxford, 2017. 
Los primeros dos capítulos son conceptuales y presentan un estudio crítico de la ira (cap. 2) y del perdón "como posible candidato a reemplazarla" (cap. 3). Allí Nussbaum plantea dos problemas internos o intrínsecos de la ira: o bien ésta busca de manera infructuosa la retribución (la "ruta del payback") y presenta problemas de incoherencia, irracionalidad e irrupciones de formas de "pensamiento mágico" que vinculan el acto de infligir sufrimiento al ofensor con la reparación del daño o la ofensa, o bien solicita la degradación del culpable (la "ruta del estatus") y es inmoral o normativamente problemática al enfocarse en forma obsesiva en el estatus y al valorar la degradación del ofensor como una oportunidad de elevar a la víctima. En este punto aparece un "tercer camino", una variedad "fronteriza" de la ira que no contiene un pensamiento retributivo: la ira transicional. Esta especie dudosa de ira sólo incluye como contenido cognitivo la indignación ante una ofensa y el pensamiento de que algo debe hacerse con ella; en este sentido, Nussbaum admite que sería mejor llamarla "indignación". Así, la ira transicional reconoce y da cuenta de una ofensa o una injusticia, y funciona como motivación para buscar la justicia. Sin embargo, a diferencia de la ira, se orienta hacia el futuro y delimita lo que está en juego no como una cuestión de restablecer límites y rangos entre el ofensor y la víctima, sino de producir justicia y cooperación.

La segunda parte desarrolla y pone a prueba las proyecciones del análisis conceptual en cuatro ámbitos de las interacciones humanas: i) el de las relaciones íntimas y personales (cap. 4); ii) el "terreno intermedio", un ámbito heterogéneo que abarca interacciones casuales con extraños y relaciones más estables que implican cierta interdependencia, es decir, relaciones con colegas, relaciones laborales y asociaciones (cap. 5); iii) el ámbito político de la "justicia cotidiana" (cap. 6); y, finalmente, iv) el espacio político de la "justicia revolucionaria" (cap. 7). En esta segunda parte, Nussbaum examina tres ideas sobre la ira que considera "lugares comunes" tanto en el pensamiento filosófico como en el cotidiano: la ira es necesaria para proteger la dignidad y el autorrespeto, la ira como reacción ante una ofensa es esencial para tomar al ofensor en serio (no como alguien con responsabilidad disminuida) y la ira es esencial para combatir injusticias. Aquí la exploración del funcionamiento de la ira en dominios diversos de la vida no busca expandir o profundizar sus problemas intrínsecos, sino discutir sobre todo sus efectos instrumentales positivos: aun si se acepta un valor limitado de la emoción para combatir injusticias e impulsar la acción política, a fin de cuentas resulta problemática en lo político y mina las bases de la comunidad igualitaria. La "ira transicional" toma como figuras centra- 
les e índices de la posibilidad y viabilidad de este modelo en el terreno político a Martin Luther King en Estados Unidos y a Nelson Mandela para el caso sudafricano.

Esta nota crítica se centra en dos aspectos del planteamiento de Nussbaum que tienen implicaciones importantes para su obra y, de manera más general, para el ámbito de la filosofía política y moral liberal. La primera se refiere al valor de las emociones y, sobre todo, al esquema de las emociones políticas que nuestra autora empezó a definir en El ocultamiento de lo humano, de 2004, y luego, de manera más explícita, en Emociones políticas, de 2013. En el contexto de la obra de Nussbaum, la ira había tenido una presencia intermitente y un estatus inestable, pero sólo hasta las conferencias John Locke de 2014 se convirtió en un foco de interés y análisis sistemático. Por otro lado, la emoción fuerte de la ira ha sido uno de los impulsos críticos a su propuesta de una cultura política que se centre en el poder ético y político de las emociones "humanizadoras" - la compasión, la simpatía-, también descritas como "emociones soft" o enmarcadas en una "política sentimental" que desactiva los espacios de resistencia y termina por privatizar la política. El nuevo eje de la ira reacomoda varias piezas del entramado que propone Nussbaum y señala continuidades y reformulaciones en su obra, a la vez que permite revisar las críticas a su propuesta filosófica.

Emociones políticas: Anger and Forgiveness en el proyecto filosófico de Nussbaum

A pesar de que Nussbaum puede considerarse una autora fuerte, una "maratonista académica", "impaciente e incluso impetuosa", extremadamente asertiva y letal con sus adversarios (Harpham 2002, p. 56), es también una filósofa del cambio y que de manera constante reformula sus ideas. Una de las formas de leer el libro comentado es situándolo en lo que ella llamó no su obra, acabada y cerrada, sino su "proyecto filosófico" que ha mutado desde los años ochenta, cuando inició su carrera, hasta la actualidad.

\footnotetext{
${ }^{2}$ Nussbaum misma ha comparado su trabajo con una maratón en diversas ocasiones. Una de las más difundidas puede leerse en el prefacio de Las mujeres y el desarrollo humano, que se considera la elaboración más completa de su conocido "enfoque de las capacidades". Allí, la autora señala que ese libro "es, en relación con aquel otro libro [un proyecto de amplio alcance sobre las capacidades que responda a todas las cuestiones técnicas, económicas y problemas políticos que presentan] como una carrera de 10 kilómetros respecto de una maratón: es completo en sí mismo, tiene un comienzo y un fin, pero no requiere tanta resistencia del lector ni del autor" (Nussbaum 2002 [2000], p. 16).
} 
Anger and Forgiveness forma parte de la obra de Nussbaum que comienza tras el cambio de milenio, con la publicación de El ocultamiento de lo humano y, sobre todo, de Emociones políticas. El proyecto que configuran estos últimos, junto con el reciente análisis de la ira, intenta, como expresó ella misma, "ligar la psicología moral de la compasión [...] con la filosofía política normativa del enfoque de las capacidades" (Nussbaum 2014 [2013], pp. 545). Estos trabajos son también un intento por salvar la brecha que suele advertirse entre una primera etapa de su obra (sobre todo la de los años ochenta) que se dedicó a la moralidad personal en un marco aristotélico que une las preguntas sobre la vida buena con las de la justicia y las estructuras que sostienen la vida en común y que apoya en dos figuras contemporáneas - Bernard Williams e Iris Murdoch-, y una segunda etapa (durante los años noventa) que se orienta hacia la filosofía política, concede un lugar más importante a Kant y vuelve prioritarios los grandes marcos institucionales y los acuerdos finos del liberalismo político en detrimento de la "percepción" densa, contextual y detallada de su primera etapa. El giro de las emociones hacia la escena política reintroduce en la segunda etapa de su obra — que había dado más espacio a la autonomía y a los límites entre las personas- a la vulnerabilidad constitutiva de la humanidad, la cual había abordado durante los años ochenta y a la cual considera uno de los "hilos" que permite unir sus preocupaciones diversas. Las emociones tienen como contenido mínimo y común el reconocimiento del valor de bienes externos que, al estar fuera del control de las personas, las vuelven vulnerables y, en este sentido, "la idea de la vulnerabilidad está estrechamente relacionada con la idea de emoción, [1] as emociones son respuestas a estas áreas de vulnerabilidad" (Nussbaum 2006 [2004], p. 19). Para Nussbaum, esa vulnerabilidad es "inseparable de nuestra sociabilidad" (Nussbaum 2006 [2004], p. 20) y desplaza la atención, más que al modo en que se trazan y se protegen las fronteras entre los individuos, hacia los modos en los que éstos se relacionan, al valor de esas relaciones y a las formas adecuadas para sostenerlas. El análisis del amor y la discusión radical que se ofrece en Emociones políticas sobre la necesidad de varios tipos de amor, confianza y reciprocidad para sostener las instituciones políticas en sociedades que aspiran a la justicia también pueden leerse en este sentido como una preocupación diferente de la que obsesionó al liberalismo por el trazado y sostenimiento de límites ${ }^{3}$ - y también a la de sus críticos

\footnotetext{
${ }^{3}$ Sobre la presencia abrumadora de la metáfora de los límites y las fronteras en la teoría política liberal, véase Nedelsky 1991.
} 
aferrados en construir una teoría del poder-, que incluye un balance de las formas que adoptan y sostienen las relaciones y un intento por desarrollar una teoría de la "fraternidad" y de los apegos. ${ }^{4}$ En este contexto, Anger and Forgiveness completa una de las obras pendientes sobre la cultura emocional de las sociedades igualitarias, y evalúa cuál es lugar que la ira debe tener en ellas y cuáles son sus alternativas, si es que deben buscarse.

Por otro lado, el estatus de la retribución y de sus pasiones asociadas como la ira es un tema en particular inestable a lo largo del proyecto filosófico de nuestra autora. La ira figuraba entre sus preocupaciones secundarias desde sus primeros libros; aparece como uno de los motivos que trataron los epicúreos y que obsesionaron a los estoicos en la reconstrucción que ofrece de las escuelas helenísticas en La terapia del deseo (1994). ${ }^{5}$ También había cuestionado la simetría de la justicia retributiva con sus pasiones asociadas en "Equity and Mercy" (1993). ${ }^{6}$ Hasta mediados de los años noventa, la ira no aparecía en su trabajo sobre las capacidades (Nussbaum y Glover 1995) pero, a partir de una sugerencia de Catharine MacKinnon, Nussbaum comienza a incorporarla durante la segunda mitad de los años noventa en la quinta capacidad de su lista como la "capacidad de sentir ira justificada" y que debía de recibir apoyo (al igual que el tipo de asociaciones que permiten su desarrollo). A la par de esa incorporación, en trabajos feministas del mismo periodo la ira se califica de "constructiva", como una contraparte del miedo constante y excesivo que la violencia ejerce sobre la vida de las mujeres, una forma de despertarlas a la rebelión y una de las emociones que deberían generar y movilizar a los grupos de mujeres, entre otros movimientos de colectivos oprimidos (Nussbaum 2002 [2000], p. 164 y Nussbaum 2005, p. 172).

Podemos destacar dos cuestiones en este repaso breve y parcial del proyecto de Nussbaum en relación con su último libro sobre la ira. Por un lado, su trabajo sobre las emociones y las "emociones políticas" brinda un contexto para leer Anger and Forgiveness y ubicarlo en el marco de un proyecto moral que reconoce el valor de las emociones e intenta

\footnotetext{
${ }^{4}$ La fraternidad, que figuraba entre los estandartes de las revoluciones liberales del siglo XVIII, fue abandonando la escena en forma progresiva a favor de los otros dos valores que la acompañaban, la libertad y la igualdad. Si bien tampoco aparecía en el vocabulario de Nussbaum, en Emociones políticas se convierte en la clave que permite leer el cambio del régimen absolutista a una sociedad igualitaria (Nussbaum 2014 [2013], p. 52).

${ }^{5}$ La ira aparece en los capítulos 7 (sobre Lucrecio), y 11 y 12 (sobre Séneca).

${ }^{6}$ El mismo desarrollo aparece luego en Sex and Social Justice (1999).
} 
emplearlas para sostener el proyecto político normativo del enfoque de las capacidades. Si las dos primeras obras de ese proyecto se dedicaron a construir un argumento a favor de la importancia de la compasión y del amor, y cuestionar el lugar de la vergüenza y el asco, la crítica a la ira que ahora se presenta parece encajar de manera intuitiva en ese esquema e incluso contribuir a apoyarlo. Por otro lado, la capacidad de "sentir ira justificada" y el espacio cada vez mayor que ocupa en el enfoque de las capacidades parecen ubicarla como parte de la idea política de ser humano en la que se basa la perspectiva. Esta última intuición es poderosa; la ira parece tan humana que un mundo que la erradique podría requerir seres muy distintos a los que somos. Nussbaum está consciente de esta objeción en Anger and Forgiveness, y se pregunta si una reacción desprovista de ira ante las ofensas no dejaría de ser totalmente humana, si no sería algo demasiado distante de lo que somos o más propia de un "santo" (pp. 105, 224-225). Gran parte de su respuesta se relaciona con las figuras de Mandela y King, que discute en los capítulos finales, cuya discusión se ubica en el "ámbito político". Ambos se presentan como pruebas no sólo del carácter humano de las reacciones que no involucran ira, sino también de su fuerza política. Sin embargo, aun si se acepta la fuerza ejemplar de ambos -en ellos la ausencia de ira no conlleva un distanciamiento inhumano; en ambos el movimiento político triunfa sin ira-, no resulta claro que puedan funcionar como ejemplos cotidianos. Ambos —como también Gandhi, su otro ejemplo- mantenían la ira bajo control mediante un sistema de creencias metafísicas difíciles de proponer en una sociedad pluralista; ambos, y sobre todo Mandela, pasaron un largo tiempo cultivando la disposición generosa que reemplaza a la ira (con un autoexamen prolongado en prisión, en el caso de Mandela). La filósofa norteamericana no sostiene que la virtud sea un camino que no requiere esfuerzo, como otros tantos objetivos que valoramos de manera cotidiana en nuestras vidas, y esto es razonable. La pregunta que queda es si ese esfuerzo no nos devuelve una imagen demasiado diferente al tipo de seres que somos, y si estamos dispuestos a pagar ese costo.

La política de las emociones: la ira y las "emociones humanizadoras"

Los casos de King y de Mandela también abren paso al argumento político sobre la ira. Aquí surge la reconsideración que Nussbaum propone de la llamada "ira noble", la ira de los oprimidos que reaccionan frente a la injusticia estructural. Este punto retoma en parte las razones por las que MacKinnon había sugerido su inclusión en el trabajo de Nuss- 
baum, y también las que ella misma adoptó al trabajar con mujeres en situación de violencia. La ira es una señal que reconoce una ofensa y una situación de injusticia; es también un poderoso catalizador de la acción y una forma de reivindicar la igualdad frente a los opresores y de cambiar o contrabalancear la situación de opresión realzando la capacidad de actuar y el sentido de dignidad de los oprimidos. Esto también se suele señalar en los estudios culturales, en algunos feminismos y en los estudios críticos sobre la raza, en relación con su propuesta de una sociedad compasiva que cultiva las "emociones humanizadoras". Lauren Berlant, quizá la más representativa de los dos primeros grupos, señala dos conjuntos de cuestiones que ponen en jaque a las emociones humanizadoras de Nussbaum: i) dan forma a un "ideal pasivo" que "transforma el imperativo ético de la transformación social [en] un ideal de compasión pasivo y de vagos tonos cívicos", donde "lo político como espacio de actos orientados hacia lo público es sustituido por un mundo de pensamientos" (Berlant 2011, p. 73); ii) al focalizarse en el entendimiento compasivo y en lo personal, esa "privatización sentimental de la política" deja inalteradas las estructuras de dominación y la posición de complicidad de quien empatiza, e incluso las sostiene y las refuerza (Berlant 2011). A ella se suman los críticos de la raza y los poscolonialistas que enfatizan la relación jerárquica que conlleva la compasión, y la amenaza de proyección y colonización del "otro" que ello implica (Delgado 1996).

Tras estas críticas puede reconstruirse una jerarquía implícita de las emociones en la que la ira figura en la cima. Así, Berlant sitúa la esperanza del cambio político en "la ira política, un discurso de demanda y crítica radical" (Berlant 1999, p. 83) y Richard Delgado (Delgado 1996) propone reemplazar la empatía por una "política del traidor a su raza" y una "subversión desde dentro". La noción de ira que parecen adoptar estos críticos es la que Nussbaum se propone desmantelar, la "ira noble", y, en este sentido, Anger and Forgiveness funciona tanto como un trabajo que completa su estudio sobre las emociones políticas, un replanteamiento de algunas áreas de su obra, pero también como una respuesta a las críticas más profundas al corazón de su propuesta sobre el valor de las emociones humanizadoras. Si bien aquí la dirección y la forma que toma esa respuesta —un análisis completo, de amplio rango y sofisticado de la ira - se presenta como el camino adecuado y valiente para defender su proyecto, sus críticas a la ira pueden resultar discutibles por el carácter "radical" que Nussbaum destaca en su propuesta (pp. 31, 44-45): en Anger y Forgiveness no sólo se discute la prioridad de la ira como catalizadora de la acción política, sino que se pretende 
que "los lectores lleguen a ver la irracionalidad y la estupidez de la ira" (p. 249) y erradicar esta emoción extendida en la vida social por completo.

El ataque contra la nobleza de la ira en situaciones de opresión es casi por completo pragmático y se centra en los casos de Gandhi, King y Mandela, quienes integran un "archivo [que] ayudará a ver por qué la idea de 'ira noble' como señal, motivación y expresión justificada es una guía falsa en situaciones revolucionarias; y por qué un marco de generosidad es más apropiado y más efectivo" (p. 212).

En los tres casos Nussbaum subraya con cuidado que ninguno de esos líderes puede considerarse naïve (o ingenuo) —un rótulo que se le ha adjudicado en ocasiones a ella misma-, y también distingue la ausencia de ira de la no violencia: la ausencia de ira es más duradera que la proclama de la no violencia, pues esta última se extingue cuando la oportunidad para la reacción violenta se agota; la ausencia de ira es crucial para la construcción de un mundo nuevo, mientras que la no violencia es sólo reactiva, genera una abstención de hacer algo en un momento determinado. La erradicación de la ira como respuesta a la injusticia tampoco implica la eliminación absoluta de la violencia. $\mathrm{Si}$ bien la violencia no es la alternativa preferida en el marco de las reacciones no iracundas, la propuesta de Gandhi "de enfrentar a Hitler sin violencia y con amor era simplemente absurda" (p. 219), y King y Mandela estaban en lo cierto cuando mantenían abierta la estrategia violenta cuando (o si) las otras vías fallaban.

Los casos que estudia Nussbaum son útiles para cuestionar la jerarquía de la ira como catalizador de la acción política. Los tres casos muestran cómo, si bien la ira puede funcionar en el corto plazo, los movimientos sociales no obtienen "coherencia y sostén" a lo largo del tiempo sólo con ella; necesitan generar además lazos de solidaridad, generosidad y empatía. La confrontación que la ira propone es parte de la política, pero también lo son las relaciones, la cooperación y los lazos de solidaridad y confianza. Por otro lado, la ira tiene un papel limitado en la construcción de un mundo nuevo que reemplace a la injusticia: genera humillación al adversario o bien lo coloca en la actitud defensiva que provoca el "juego de la culpa", distrae la atención del problema principal e impide la cooperación en la construcción de nuevas comunidades incluyentes. Sin embargo, los tres casos también arrojan sombras sobre la viabilidad y deseabilidad de erradicar la ira. En primer lugar, la ira puede mantener un valor intrínseco y reivindicativo para los oprimidos a quienes por lo común se les deniega socialmente el derecho a la rabia, a la furia o al enojo. La ira no parece distribuirse de manera igua- 
litaria en la sociedad; en los varones se trata de una emoción permitida, en las mujeres es signo de exceso, irracionalidad o locura; asociada a los blancos puede considerarse un signo de autorrespeto, mientras que la ira negra se presenta como señal de una violencia innata e irracional. En esos grupos oprimidos, reivindicar la ira como respuesta a la injusticia puede tener un valor expresivo más que retributivo y no implicar de manera interna el deseo de payback como lo pretende Nussbaum. En otras palabras, la reivindicación de la ira negada para ciertos grupos puede implicar ella misma la "retribución" (en el sentido sofisticado de restituir la igualdad) ante la ofensa, sin que conlleve ni un deseo de infligir sufrimiento al ofensor (payback) ni tampoco degradar el estatus del ofensor para compensar el de la víctima: la expresión de ira es, en estos casos, una forma de nivelar y marcar puntos de igualdad entre las víctimas y los ofensores, y una forma también de redistribuir la economía social de las emociones.

Por otro lado, los casos que presenta Nussbaum no terminan de probar que la ira no sea necesaria en la política o que tenga un carácter nocivo e improductivo. En los movimientos de Gandhi, Mandela y King puede haber funcionado de ese modo, pero el argumento es pragmático y empírico, y el lector se pregunta aquí si este marco de análisis basado en un puñado de casos podría aplicarse a otras luchas de reivindicación de derechos - el caso que rápidamente viene a la mente es el de los movimientos LGBTIQ- y qué hubiera sido de ellas sin el recurso, aun momentáneo y reactivo, a la ira, la furia o la rabia pública. Se puede incluso dudar de la ausencia de ira en algunos de los casos que Nussbaum plantea como el factor determinante de su éxito político. Como señala Srinivasan (Srinivasan 2016), la estrategia de evitar la ira de Martin Luther King parece depender de la ira de Malcolm X y de las panteras negras, y sin esa ira que corría en paralelo a King puede resultar difícil explicar la reacción que tuvo el Estado a fin de cuentas. En cualquier caso, el argumento de Nussbaum aquí es más pragmático y empírico que normativo, y si bien los casos que señala marcan un camino de posibilidad para su propuesta de erradicación de la ira, no resuelven la disputa sobre su productividad política.

El libro de Nussbaum es una obra importante para leer su proyecto filosófico y abre, al igual que sus escritos anteriores, campos de investigación que permanecen aún en estado incipiente: el silencio forzoso de las emociones en la teoría política liberal, el uso de materiales literarios en principio ajenos a la pulcritud de la filosofía analítica angloamericana, el reposicionamiento de la vulnerabilidad como dimensión de la experiencia humana, la reintroducción de la importancia de la con- 
fianza en un clima dominado en la teoría crítica y en el pensamiento cotidiano por la "sospecha", la valorización de la fraternidad como ideal distintivo junto a la libertad y la igualdad. Anger and Forgiveness presenta una plataforma sólida, erudita y amplia para discutir el estatus de la ira en nuestras sociedades desiguales, rotas y que padecen altos niveles de violencia.

\section{BIBLIOGRAFÍA}

Berlant, L., 1999, "The Subject of True Feeling: Pain, Privacy, and Politics", en A. Sarat y Th. Kearns (comps.), Cultural Pluralism, Identity, Politics, and the Law, University of Michigan Press, Ann Arbor, Míchigan, pp. 49-84.

__, 2011, "Pobre Eliza", El corazón de la nación: ensayos sobre política y sentimentalismo, trad. V. Schussheim, Fondo de Cultura Económica, México, pp. 59-112.

Delgado, R., 1996, "Rodrigo's Eleventh Chronicle: Empathy and False Empathy", California Law Review, vol. 84, no. 1, pp. 61-100.

Harpham, G., 2002, "The Hunger of Martha Nussbaum", Representations, vol. 77, no. 1, pp. 52-81.

Nedelsky, J., 1991, "Law, Boundaries, and the Bounded Self", en R. Post (comp.), Law and the Order of Culture, University of California Press, Berkeley, pp. 162-184.

Nussbaum, M.C., 1999, Sex and Social Justice, Oxford University Press, Oxford.

—_, 2002, Las mujeres y el desarrollo humano, trad. R. Bernet, Herder, Barcelona [1a. ed.: 2000].

__ 2003, La terapia del deseo. Teoría y práctica en la ética helenística, trad. M. Candel, Paidós, Barcelona [1a. ed.: 1994].

_ 2005, "Women's Bodies: Violence, Security, Capabilities", Journal of Human Development, vol. 6, no. 2, pp. 167-183.

—_, 2006, El ocultamiento de lo humano: repugnancia, vergüenza y ley, trad. G. Zadunaisky, Katz, Buenos Aires [1a. ed.: 2004].

— 2014, Emociones políticas. ¿Por qué el amor es importante para la justicia?, trad. A. Santos Mosquera, Paidós, Barcelona [1a. ed.: 2013].

—_, 1993, "Equity and Mercy", Philosophy and Public Affairs, vol. 22, no. 2, pp. 83-125.

Nussbaum, M.C. y J. Glover (comps.), 1995, Women, Culture and Development, Oxford University Press, Oxford.

Srinivasan, A., 2016, "Would Politics Be Better Off without Anger?, The Nation, 30 de noviembre de 2016. 